\title{
Persistent Left Superior Vena Cava in a Patient with Congenital Heart Disease
}

\author{
Christian S. Haas ${ }^{*}, 1$ and Volker Doernberger ${ }^{2}$ \\ ${ }^{1}$ University of Luebeck, Department of Medicine I, Luebeck, Germany \\ ${ }^{2}$ University Hospital Tuebingen, Department of Internal Medicine, Division of Cardiology and Cardiovascular Medi- \\ cine, Tuebingen, Germany
}

Keywords: Persistent left superior vena cava, pacemaker, fluoroscopy, congenital heart disease.

\section{LETTER TO THE EDITOR}

A 40-year old man with a history of surgically corrected congenital infundibular pulmonal stenosis and subvalvular membranous aortic stenosis presented with symptomatic sick sinus syndrome. Sick sinus syndrome may be related to stretching of the atrioventricular node and bundle of His, often a result of changed hemodynamics due to the underlying congenital disease. Implantation of a DDDR pacemaker was initiated, but positioning of the pacemaker leads proved to be difficult while pushing the lead forward. Radiographic imaging with contrast media was performed, revealing a persistent left superior vena cava (PLSVC). In knowledge of the abnormal anatomy, placement of the atrial and ventricular leads was still challenging but could be completed using fluoroscopy without major problems. The chest radiograph after the procedure showed a left-sided path of both pacemaker leads via the PLSVC (arrowheads), with correct position of the tips in the right atrium (open arrow) and in the right apical ventricle (arrow), suggesting connection with the right atrium (Fig. 1).

PLSCV is the most common variation in the thoracic venous system and present in about $0.5 \%$ of the normal population. Its incidence is even higher in patients with congenital heart disease $(2-5 \%)$, particularly in those with pulmonary stenosis, atrioventricular septal defects and Dtransposition [1]. PLSCV usually drains into a dilated coronary sinus but may alternatively be connected to the right or left atrium [2]. Various imaging studies, including echocardiography, computed tomography and magnetic resonance tomography can help to detect this vascular anomaly [3]. However, inexperienced examiners may miss PLSCV. In the present case, PLSCV had not been known from earlier angiographic or echocardiographic studies, e.g. prior to heart surgery. A possible explanation might be the younger age of the patient at the time of surgery with less prominent features of PLSCV or the physicians approach with focus on the main congenital heart defect, thereby missing the vascular

*Address correspondence to this author at the University of Luebeck, Hospital Schleswig-Holstein - Campus Luebeck, Department of Medicine I, Ratzeburger Allee 160; 23538 Luebeck, Germany; Tel: +49-451-500-5060; Fax:+49-451-500-5066; E-mail: cs_haas@yahoo.com anomaly. In fact, even in patients with congenital heart disease, diagnosis of PLSCV is often the result of an incidental finding during diagnostic and therapeutic procedures, such as central vein placement, pacemaker lead insertion or right heart catheterization [4]. Physicians should be aware of this anomaly and possible approaches in case of unexpected problems with central venous access [5].

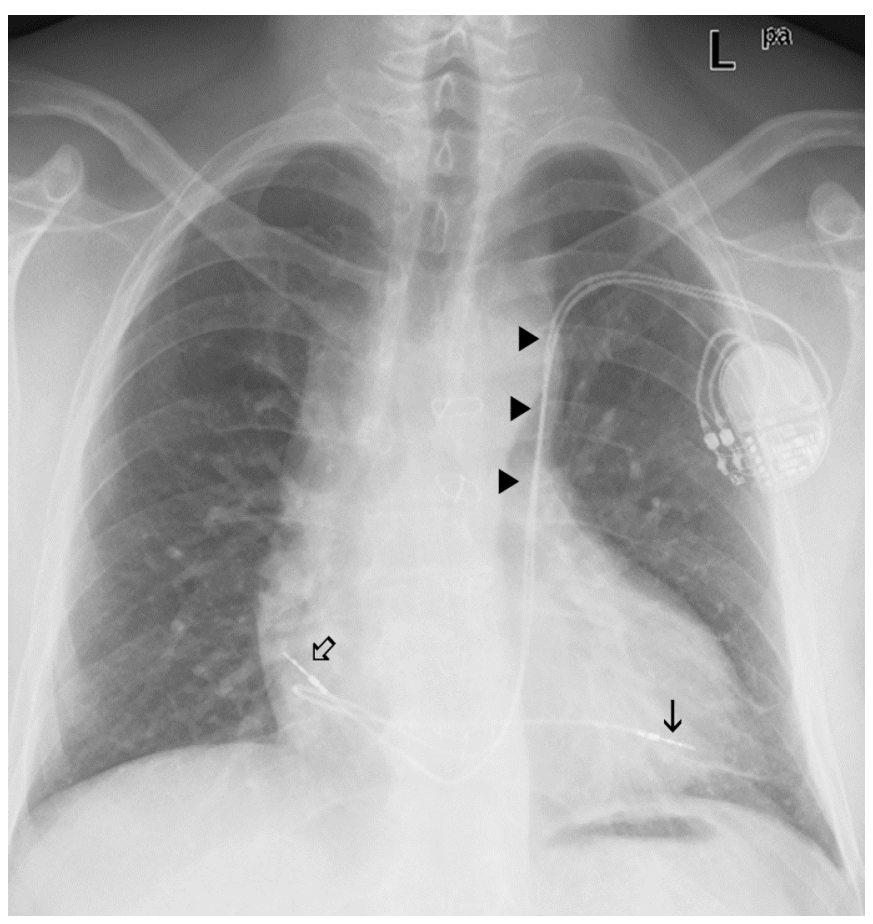

Fig. (1). The chest radiograph after the procedure showed a leftsided path of both pacemaker leads via the PLSVC (arrowheads), with correct position of the tips in the right atrium (open arrow) and in the right apical ventricle (arrow), suggesting connection with the right atrium.

\section{REFERENCES}

[1] Rigatelli G. Congenitally persistent left superior vena cava: a possible unpleasant problem during invasive procedures. J Cardiovasc Med 2007; 8: 483-7.

[2] Brickner ME, Eichhorn EJ, Netto D, et al. Left-sided inferior vena cava draining into the coronary sinus via persistent left superior vena cava: case report and review of the literature. Cath Cardiovasc Diagn 1990; 20: 189-92. 
[3] Recupero A, Pugliatti P, Rizzo F, et al. Coglitore S persistent leftsided superior vena cava: integrated noninvasive diagnosis. Echocardiography $2007 ; 24$ : 982-6.

[4] Sarodia BD, Stoller JK. Persistent left superior vena cava: case report and literature review. Respir Care 2000; 45: 411-6.
[5] Sticherling C, Osswald S. Implantation of a biventricular defibrillator system in a patient with persistent left and absent right superior vena cava. Heart 2006; 92: 1424.

(C) Haas and Doernberger; Licensee Bentham Open.

This is an open access article licensed under the terms of the Creative Commons Attribution Non-Commercial License (http: //creativecommons.org/licenses/by-nc/ $3.0 /$ ) which permits unrestricted, non-commercial use, distribution and reproduction in any medium, provided the work is properly cited. 